\title{
Howard Russell Butler: Painter Extraordinary of Solar Eclipses
}

\section{Rolf M. Sinclair}

\begin{abstract}
Howard Russell Butler (1856-1934) was a successful landscape and portrait painter who discovered a rare talent for seeing an image briefly and then painting it from memory and a few notes. He originally studied physics at Princeton, worked in the nascent telephone industry, and then practiced law. His avocational interest in painting grew until, at age 28 , he made the decision to become a professional artist. He sometimes used his unusual talent to quickly sketch transient phenomena (or a busy patron) and then later finish the painting. Since colour photography was then unable to capture the phenomena visible only during a total solar eclipse, Butler was commissioned to capture the nuances and colours of the solar corona and prominences in the precious seconds of several eclipses. His paintings became astronomical classics. He went on to paint other astronomical themes (such as Mars seen from its Moon and design a museum's Ideal Astronomic Hall, using the astronomical knowledge of his day. Although these scientific works were only a small part of his oeuvre, they mark him as one who uniquely brought together art and astronomy. This talk will show the range of Russell's works and describe his unusual techniques.
\end{abstract}

A total eclipse of the Sun is possibly the most impressive astronomical phenomenon that we can experience with the naked eye. The sky darkens as the Moon passes in front of the Sun, causing it to vanish briefly to be replaced by a black disk surrounded by pale structures of glowing gas. Such eclipses are rare, and occur only once or twice a year along a narrow path somewhere in the world. Alternatively, any one place experiences an eclipse perhaps every 300 years, on the average. As a result, few people have seen a solar eclipse. It has proven very difficult to make a good representation of what the eye sees at an eclipse, so that a larger number of people can appreciate one.

Howard Russell Butler (1856-1934) became famous in the 1920s for his paintings of astronomical subjects, in particular for his ability to make accurate paintings of the eclipsed Sun. A total solar eclipse lasts at most a few minutes at an observation site, and since it is visible only along a path a few tens of miles wide, observers must travel to the often-

Rolf M. Sinclair, 'Howard Russell Butler: Painter Extraordinary of Solar Eclipses', eds. Nicholas Campion and Rolf Sinclair, Culture and Cosmos, Vol. 16 nos. 1 and 2, 2012, pp. 345-355.

www.CultureAndCosmos.org 
remote locales where an eclipse can be seen. It is only during a total eclipse when the direct light from the Sun is cut off that the cloud of hot gases that surround the Sun (the faint solar corona) is visible to the naked eye. Butler was able to make enough notes in the brief time of totality that then enabled him to make an accurate rendition of an eclipse. These paintings were a tour de force in their day when photography could not yet capture the nuances of the eclipsed Sun, and they provided astronomers and the public with perhaps the best naked-eye record of eclipses at that time. ${ }^{1}$

Butler became an artist by following an unusual career path. He was born into a prosperous family of lawyers on his father's side and ship owners on his mother's side. He developed an early interest in art by taking private lessons from age twelve, but elected to enter the College of New Jersey (now Princeton University) in 1872 to study physics, graduating in 1876. He stayed for a further year as assistant to Professor Cyrus Fogg Brackett (1833-1915), whose work covered both physics and what was then the beginning of electrical engineering. Butler then went to work in the nascent telephone industry, and after studying at Columbia University Law School, he was admitted to the New York Bar, opening his own practice in New York City in 1882, specializing in patent law.

All the time his avocational interest in art had been with him and in 1884, at age twenty-eight, he made the bold decision to change careers and become a professional artist. He found excellent tutors, studying under Frederick Edwin Church (1826-1900) in Mexico in 1885, and then in Paris from 1886-87 under Pascal Dagnan-Bouveret (1852-1929) and Henri Gervex (1852-1929). He then returned to the United States and started his own career in art, quickly becoming known as a leading portrait and landscape painter. Recognition by his peers included his service as an official of several national professional artists' associations. $^{2}$

\footnotetext{
${ }^{1}$ The reader is referred to Jay Pasachoff, Complete Idiot's Guide to the Sun (Indianapolis IN: Alpha Books, 2003), and to Leon Golub, and Jay Pasachoff, The Nearest Star (Cambridge MA: Harvard University Press, 2001), for popular descriptions of solar eclipses and how they are studied from Earth and (more recently) from space. Leon Golub, and Jay Pasachoff, The Solar Corona (Cambridge: Cambridge University Press, 2nd ed., 2010) is a more advanced technical study of the corona.

${ }^{2}$ Butler's career and paintings are described in F. Newlin Price, Howard Russell Butler 1856-1934 (Princeton: Princeton University Press, 1934).
} 
Two events then happened to alter his career. The first, in about 1890, was his painting of a dozen or more portraits of Andrew Carnegie (1835-1919); this led Carnegie to recognize his talents by commissioning him to plan and supervise several major projects, which still allowed him to continue his career as an artist. These projects included Carnegie's New York City mansion (now the Smithsonian Cooper-Hewett, National Design Museum), Lake Carnegie at Princeton University and Carnegie Hall (New York City) where Butler served as President from 1896-1905. This made Butler known to a wide circle of art patrons.

The second event occurred in 1916 when Butler was in the act of painting a moonlit scene on the Maine coast where he spent his summers. Unexpectedly a brilliant aurora borealis flashed out, and Butler used his unusual skills at making quick visual notes of shape, hue, shade, and luminosity to capture the features of the aurora which lasted only forty minutes; he was later able to make from these notes what proved to be the first of his paintings of astronomical subjects (Figure 1). ${ }^{3}$

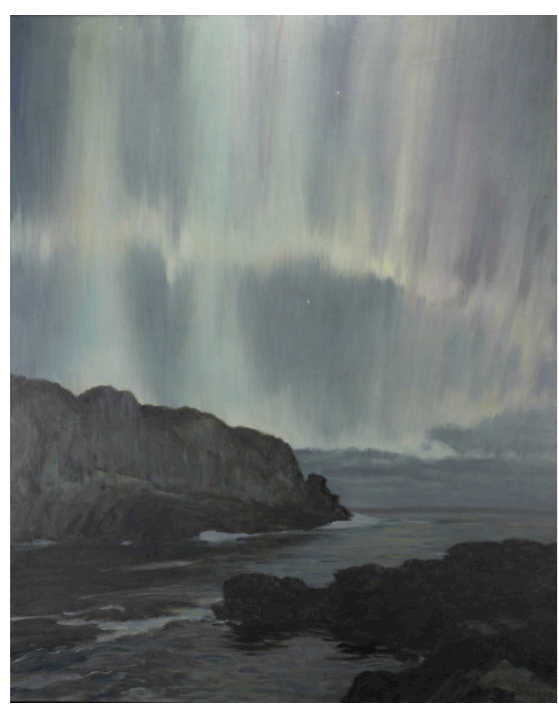

Figure 1. Howard Russell Butler, Aurora Borealis (1916) Princeton University Art Museum, by permission of the Princeton University Museum

Samuel Alfred Mitchell (1874-1960), who was then Professor of Astronomy and Director of the McCormick Observatory at the University of Virginia, had spent a number of years studying the Sun, and what could be learned by observing the occulted disk during total solar eclipses

\footnotetext{
${ }^{3}$ Anon., 'The Wonders of the Sky are Caught in Paint', New York Times Magazine, 25 April 1926.
} 
when the Moon completely covers the surface of the Sun. Throughout human history, this was the only way to observe and study the faint solar corona, when an eclipse would show, for a few minutes every year or two, the tenuous atmosphere of hot gases enveloping the Sun. The colour film then available could not capture the details and colours of the corona during the precious seconds of totality, and drawings made of eclipses were not sufficiently accurate. In 1923, Mitchell wrote:

What was perhaps the most interesting piece of scientific work accomplished at the 1918 eclipse owes its conception to Mr. Edward D. Adams [(1846-1931); an industrialist and amateur astronomer, who served as a trustee of the Metropolitan Museum of Art, New York City from 1894 to 1931.]... Upon becoming a member of the US Naval Observatory party Mr. Adams took upon himself the responsibility of trying, by some method, by photography, by a drawing, or by a painting, to procure a reproduction which would show the beauties of the corona, and which should be true not only as to form but more especially as to colour. Unfortunately for science it is impossible to obtain a satisfactory representation of the corona...by photography...The eye can take cognizance of the details in spite of the great changes in brilliance, but not so the photographic plate... Mr. Adams took upon himself the task of finding the right man... who would have the true scientific spirit and who could combine an accurate sense of form with a refined perception of colour. [He] was successful in finding $\mathrm{Mr}$. Howard Russell Butler, a portrait painter of note, who has developed a shorthand method of noting both form and colour. ${ }^{4}$

Butler joined the 1918 USNO party in Oregon, and after some weeks of study laid out a scheme in advance for painting the eclipse, from which he produced a remarkable painting (Figure 2).

4 S.A. Mitchell, Eclipses of the Sun 1st ed. (New York City: Columbia University Press, 1923), pp. 204-05. Later editions of this book contain more information on the 1923 and 1925 eclipses. See also S. A. Mitchell, Eclipses of the Sun, in G. Eberhard et al. (eds.), Handbuch der Astrophysik Band IV Das Sonnensystem (Berlin: Verlag von Julius Springer, 1929), p. 254.

Culture and Cosmos 


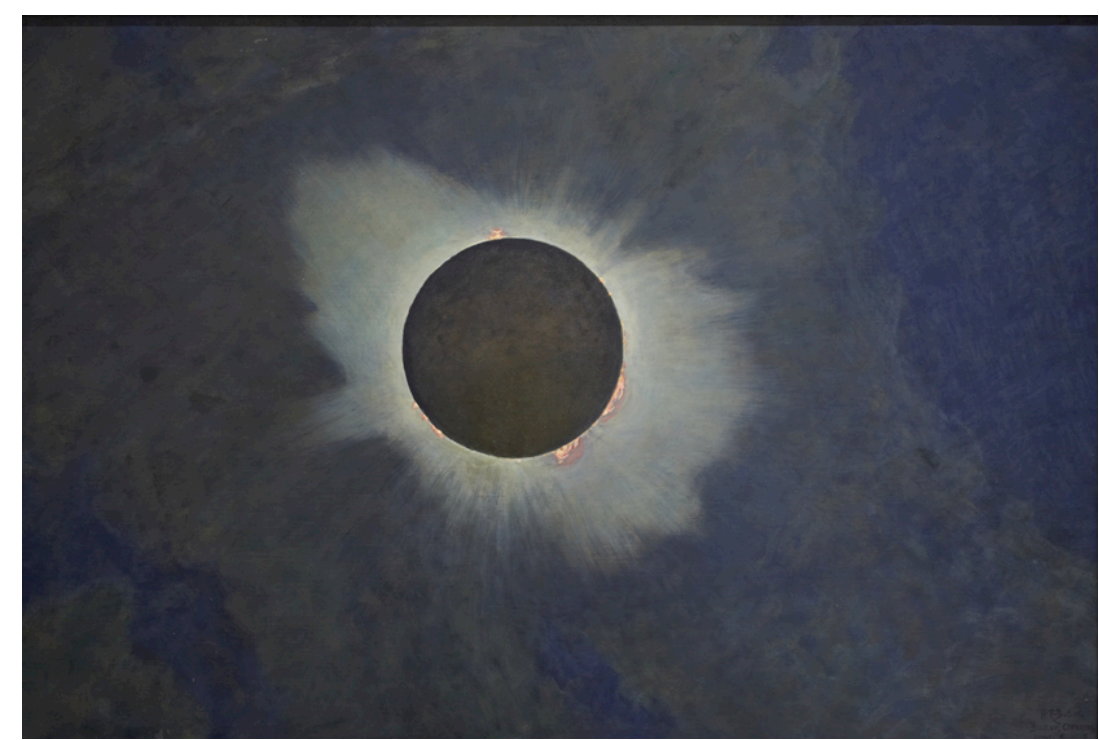

Figure 2. Howard Russell Butler, Solar Eclipse, Baker, Oregon, June 8, 1918 (1918) Princeton University Art Museum, by permission of the Princeton University Museum.

Mitchell went on to write: 'The astronomers who saw the 1918 eclipse and who have seen [Butler's painting] look upon it as...true both as to form and colour, a great work of art which has the added advantage of being scientifically accurate. The world of science owes a great debt of gratitude to Mr. Butler... ${ }^{5}$ It is worth noting that Butler had never seen a total eclipse of the Sun prior to this. He once accompanied a group from his College to see an annular eclipse in New Hampshire in 1875, which however did not allow him to see the corona. ${ }^{6}$ (The Moon's apparent size varies over time, from slightly larger than the apparent size of the Sun to slightly smaller. If an eclipse occurs when the Moon appears larger than the Sun, it can cover the Sun's disk; the eclipse is total, and the corona can appear. If however the Moon appears smaller than the Sun, the eclipse is not total; rather, a thin ring at the edge of the Sun still shows around the Moon, and the eclipse is called annular. Even this small amount of visible solar surface is enough to blot out the much fainter

\footnotetext{
${ }_{6}^{5}$ Mitchell, Eclipses 1923, p. 212.

${ }^{6}$ Elizabeth Stevens, Howard Russell Butler. An exhibition of his oils, pastels, and drawings (the catalog of an exhibit at Squibb Gallery, Princeton NJ, privately printed, 1937).
} 
corona, which then cannot be seen.) There were no total eclipses visible from the US between 1869 and 1878, perhaps making an annular eclipse worth travelling to see. Butler later wrote in 1926:

When asked if I would accompany the USNO party to Baker, Oregon, and try my hand at painting the solar eclipse of June 8, 1918 , I stated that, as a portrait painter, I generally asked for 10 sittings of 2 hours each. But all the time they would allow me on this occasion was $1121 / 10 \mathrm{sec}$. As it turned out I got a trifle more, for their calculations were short by $3 / 10$ of a second.

Of course no actual painting was possible in time so limited and I therefore employed a method of shorthand sketching (which I had been developing and practicing for about 20 years) for recording sunsets and other transient effects. The method is simple but does require practice. ${ }^{7}$

Using these notes, Butler could in the next hours paint the eclipse while it was fresh in his memory, becoming the first person to make a painting directly of a particular solar eclipse that showed accurately what an observer would have seen. Butler describes his method in detail in two publications. ${ }^{8}$ In essence, he took the shorthand notation method he had developed for painting sunsets and other transient phenomena, and developed a condensed version for eclipse painting that he called a 'shorthand of a shorthand method'. He started with an approximate outline of what to expect, and then allotted certain times (measured in seconds) to sketch the outlines of various features and to note their colour ranges. He allowed a little time to examine the corona and solar prominences through field glasses. He made perhaps ten practice runs before the eclipse. Then as the actual eclipse was approaching, he carefully looked away from the Sun at the darkening sky, allowing his eyes to accommodate. When an astronomer shouted 'Go!', he turned to view the eclipsed Sun with dark-adapted eyes. An observer then counted the seconds to keep him on schedule. Once the eclipse ended he started on a painting, and then the next day a second. A third was later executed

\footnotetext{
${ }^{7}$ Howard Russell Butler, 'Painting Eclipses and Lunar Landscapes'. Natural History Vol. XXVI, No. 4, July/August 1926, p. 356.

${ }^{8}$ Howard Russell Butler, 'Painting the Solar Corona. Natural History Vol. XIX, No. 3, March 1919, pp. 264-272; Butler, 1926, pp. 356-362.
} 
using photographs of the prominences and coronal structure to help fill in the details. Even with these aides mémoires, he had to rely on his notes made during the eclipse for the colours of these features.

Butler went on to join the USNO eclipse expeditions of 1923 and 1925, and painted those eclipses. He noted that the later eclipses were easier to paint since he had a much better idea of what to expect. Interestingly, for the 1923 eclipse the USNO team went to San Diego with other observing parties to use the facilities of Fort Rosecrans Military Reservation. Unfortunately the weather was not cooperative, and all they saw that day was the underside of rain clouds. For some reason Butler elected to go further up the coast near Santa Barbara, where he had a good view of the eclipse and could record it. In 1925, he again joined the astronomers, this time in Connecticut. After painting three eclipses he went on to paint several triptychs depicting all of them. The triptychs at the Franklin Institute and at the Buffalo Museum of Science are still on display. A third was painted for the American Museum of Natural History; although that one is no longer on display a copy has been published. $^{9}$

A curious thing happened in 1932. There was an eclipse that year, but this time it came to Butler rather than vice-versa. For a number of years Butler had a summer house and studio in York Harbor, Maine, where he could paint his beloved Maine coast; he painted the famous 'Aurora' subject at Ogunquit, about eight miles to the North. It so happened that his house was within the path of the eclipse, close to the centre of totality, so he could paint this eclipse from the comfort of his own studio. ${ }^{10}$

In the 1920s Butler was active at the American Museum of Natural History as an artistic consultant on things astronomical. As well as seven large panels illustrating solar prominences, he painted several lunar landscapes with the Earth high in the sky. These moonscapes were a joint project between Butler and Henry Norris Russell (1877-1957), then the Professor of Astronomy at Princeton. The idea for a 'lunar landscape' had occurred to Butler earlier, and at an evening party in Princeton he mentioned it to Russell, who offered on the spot to help him, and they proceeded to the Princeton Observatory to study the Moon's surface. After some work they found a desirable place centred on a minor crater

\footnotetext{
${ }^{9}$ This triptych is illustrated in the Supplement to Natural History, Vol. XXVI, July/August 1926.

10 'Eclipse of Sun Edition', Science News Letter, Vol. XXII, No. 590, July 30, 1932, pp. 62-76.
} 
surrounded by mountains. ${ }^{11}$ The completed picture is a view of the Earth as seen from that crater, incorporating various features of this actual place on the Moon (Figure 3).

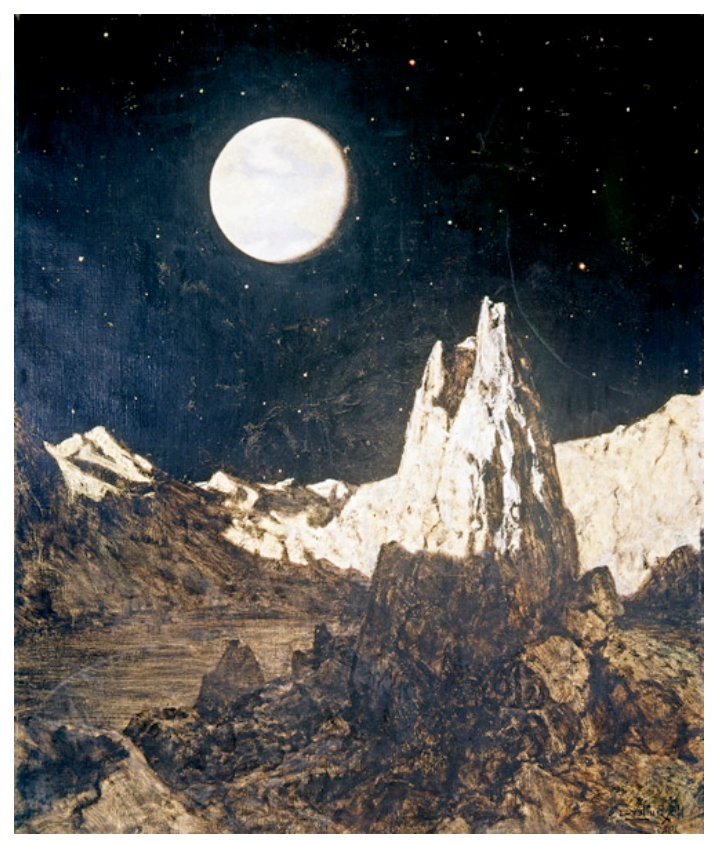

Figure 3. Howard Russell Butler, Lunar landscape (ca. 1926), by permission of the American Museum of Natural History

Every effort was made to portray the Earth correctly in the starry sky, as it would be seen from the Moon in the month of June. ${ }^{12}$ Butler's painting of a craggy lunar landscape set the style for illustrations of scientific and science-fiction articles for several decades. [This particular landscape, which was imaginary but plausible at the time, probably owes a lot to Butler's paintings done about that time in Zion and Bryce National Parks. ${ }^{13}$ ] Butler also made several paintings of Mars as seen from its moons (Figure 4). Here again he worked with astronomers at several observatories who helped him to form an image of what Mars might look like at close range.

\footnotetext{
${ }^{11}$ Anon., Wonders, 1926.

${ }^{12}$ Butler, 1926, pp. 361-62.

${ }^{13}$ Price, 1934.
}

Culture and Cosmos 


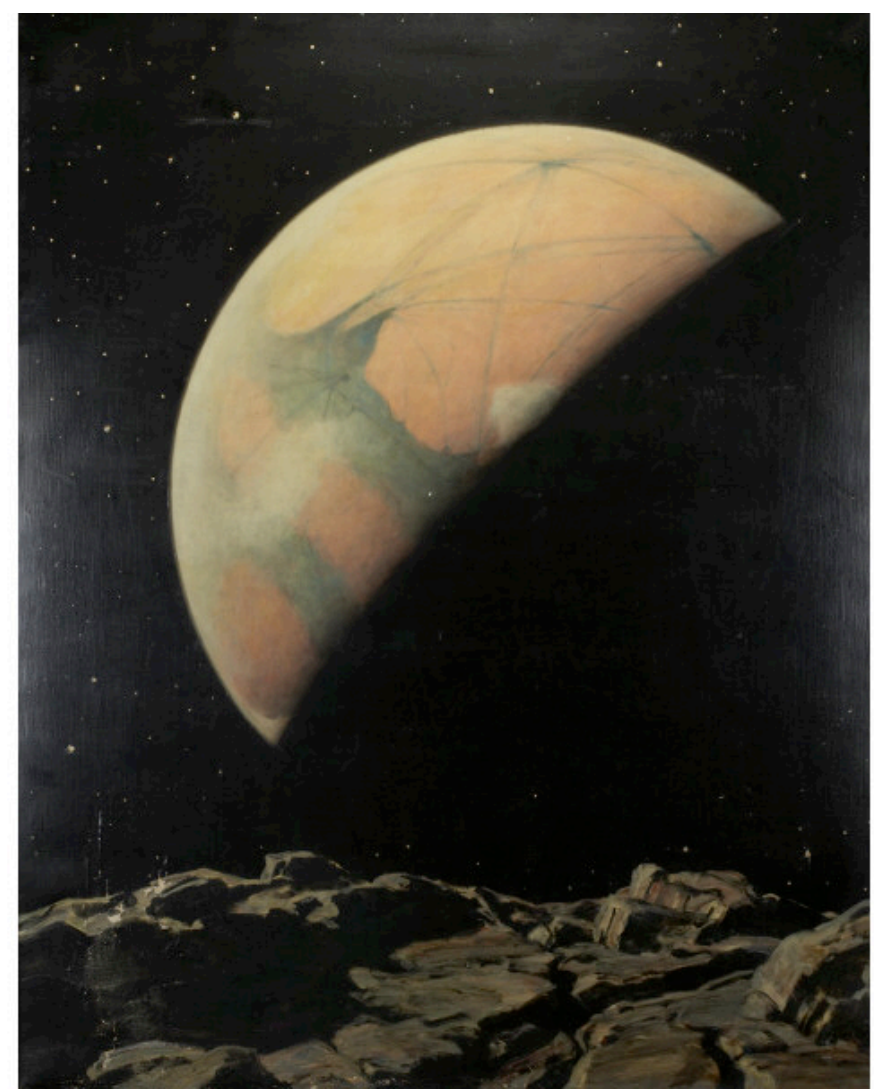

Figure 4. Howard Russell Butler, Mars seen from Deimos (ca. 1926) Princeton University Art Museum, by permission of the Princeton University Museum

Butler went on to design an 'Astronomic Hall' which would have been a central feature of the Museum featuring astronomical exhibits on three floors, with a planetarium on the top floor. ${ }^{14}$ The project was seriously considered, but the funds could not be found and the Hayden Planetarium was instead constructed in a separate building. However, there were displays of his artwork featured in the Museum and in the Hayden

${ }^{14}$ Howard Russell Butler, 'An Ideal Astronomic Hall', Natural History Vol. XXVI, No. 4, July-August 1926, pp. 392-98; Jenny Lawrence and Richard Milner, 'A Forgotten Cosmic Designer'. Natural History, February 2000.

Culture and Cosmos 
Planetarium for a number of years, serving to "stir the imagination of several generations of schoolchildren'. ${ }^{15}$

What can we say about the importance of Butler's astronomical paintings? Through the centuries many artists have painted works that depict solar eclipses. Those done before the late nineteenth century tended to be more symbolic than realistic portrayals. Since the late nineteenth century other artists produced realistic renderings of solar eclipses, but none approach Butler's in care and accuracy. Butler's eclipse paintings remain unique, and will probably never be equalled. They show an unusual use of artistic talent to solve a problem in astronomy. They were important at the time as aids to astronomers, and were particularly popular with the general public. These and his other astronomical paintings played an important role in making the new findings in astronomy available to students and to the public.

Visual depiction of astronomical events has progressed far beyond what was possible in Butler's day. The introduction of new types of telescopes and the ability to observe from space stations have altered astronomy profoundly. For example, the last decades of solar studies have revolutionized our understanding of the Sun and show us that the earlier ground-based eclipse studies of the solar corona were only a twodimensional projection of very complex three-dimensional phenomena. ${ }^{16}$

Now that humans have reached the Moon and automatic stations have reached Mars to return detailed studies, we realize that as long as we were limited to ground-based, naked-eye, telescopic studies we could not have anticipated the reality of the lunar and Martian surfaces. Thus, Butler's moonscapes were too rugged and dramatic compared to the rather dull reality found by the Apollo astronauts. Butler's views of Mars also showed the limits of naked-eye observations, which could not resolve what we now know to be deep valleys and craters, but rather tended to suggest quasi-artificial linear features. Butler's works served in the development of astronomy and were in turn superceded by sophisticated visual technologies. Nevertheless, they have a place in the history of astronomy in the first half of the twentieth century.

Butler lived in Princeton for several decades, and there he died in his home in 1934. He has a modest memorial in The Princeton Cemetery, marked only with his name, dates, and an artist's palette. His obituary in

\footnotetext{
${ }^{15}$ Lawrence, 2000.

${ }^{16}$ Richard Woo, 'Revealing the True Solar Corona'. American Scientist Vol. 98, May/June 2010, pp. 212-19.
} 
the New York Herald Tribune indicated that he was best known at that time for his paintings of astronomical subjects, calling him a 'Painter of Sun and Stars' who 'Put Solar Eclipses in Oil' and 'Pictured Earth from Moon and Heavens from Mars' ${ }^{17}$

\section{Acknowledgements.}

I first became aware of Butler's astronomical paintings through a chance encounter as an undergraduate with Mitchell's Eclipses. My more detailed study of Butler's works started in the 1970s, when Doreen Bolger (Baltimore Museum of Art [then at the Metropolitan Museum of Art]) sent me a wealth of information on his life and paintings. This material proved essential in preparing the present paper, which owes its genesis to the inspiration of the INSAP conferences. I am particularly grateful for the invaluable help of Lisa Arcomano (Princeton Museum), and of Neil deGrasse Tyson and Mai Qamaran (AMNH), in locating and making accessible many of Butler's astronomical paintings. The Franklin Institute, the Buffalo Museum of Science, the Museum of Science (Boston), and the Staten Island Museum were most cooperative in locating their Butlers and sending me photos. Gino Francesconi (Director, Archives and Museum, Carnegie Hall) told me the interesting story of Butler's interactions with Andrew Carnegie.

\footnotetext{
${ }^{17}$ Anon., 'H. R. Butler, 78, Painter of Sun and Stars, Dies'. New York Herald Tribune, March 23, 1934. The best collections of his astronomical paintings are in the Princeton University Art Museum and in the American Museum of Natural History. Price (1934) gives the location of many of his works, which are scattered among several museums and many private collections. The largest number of his non-astronomical works is at the Smithsonian American Art Museum which has some 60 of his paintings and sketches.
}

Culture and Cosmos 